\title{
Clinical experience and critical evaluation of the role of everolimus in advanced renal cell carcinoma
}

\author{
Maxine Sun' \\ Firas Abdollah ${ }^{2}$ \\ Jan Schmitges' \\ Claudio Jeldres' \\ Shahrokh F Shariat ${ }^{3}$ \\ Paul Perrotte ${ }^{4}$ \\ Pierre I Karakiewicz 1,4 \\ 'Cancer Prognostics and Health \\ Outcomes Unit, University of \\ Montreal Health Center, Montreal, \\ Canada; ${ }^{2}$ Department of Urology, Vita \\ Salute San Raffaele University, Milan, \\ Italy; ${ }^{3}$ Department of Urology, Weill \\ Medical College of Cornell University, \\ New York, NY, USA; ${ }^{4}$ Department \\ of Urology, University of Montreal \\ Health Center, Montreal, Canada
}

This article was published in the following Dove Press journal:

Open Access Journal of Urology

2I April 20I I

Number of times this article has been viewed

\begin{abstract}
The efficacy of sequential everolimus, an orally administered inhibitor of mammalian target of rapamycin (mTOR), was proven in a placebo-controlled phase III study, where median progression-free survival was 4.9 vs 1.9 months for placebo (hazard ratio: $0.33, P<0.001$ ). Placebo crossovers $(80 \%)$ contaminated overall survival data. Adverse event discontinuation rate was of only $10 \%$ and health-adjusted quality-of-life was sustained. These data represent the first placebo-controlled evidence of efficacy for a sequentially used targeted agent. Everolimus resulted in the strongest hazard ratio ever recorded for progression-free survival, despite it being tested in a population with the most aggressive natural history ever recorded in all available phase III metastatic renal cell carcinoma trials. Everolimus use after exclusively one prior antivascular endothelial growth factor failure resulted in an even longer progression-free survival time (5.4 months) than in the entire population (4.9 months). These benefits should also be considered in the light of sustained and unimpaired health-related quality of life. Use in first line other than second or subsequent lines remains to be validated.
\end{abstract}

Keywords: everolimus, metastatic renal cell carcinoma, targeted therapy, sequential therapy, mTOR

\section{Introduction}

Renal cell carcinoma (RCC), predominantly of clear cell histological subtype (85\%), is associated with a loss of function of the von Hippel-Lindau (VHL) gene on chromosome 3p. Defects in VHL are the most common cause of inherited clear cell RCC, and are characterized by an upregulation of hypoxia-inducible factor (HIF). The downstream molecular effects of VHL-deficient cells involve the accumulation of the angiogenic growth factors vascular endothelial growth factor-A (VEGF-A), plateletderived growth factor- $\beta$ (PDGF- $\beta$ ), and transforming growth factor- $\alpha$ (TGF- $\alpha$ ), as well as a corresponding increase in angiogenic factors.

Previously, metastatic RCC (mRCC) patients' only effective therapies consisted of high-dose interleukin-2 or interferon- $\alpha$, with little, if any, improvement in survival. More recently, several agents have been introduced in the treatment paradigm of patients with mRCC (Table 1). Sunitinib and sorafenib target the angiogenic response by inhibiting the ability of endothelial cells to respond to the overproduction of angiogenic growth factors VEGF and PDGF. A previous phase III trial $(n=750)$ showed that sunitinibtreated patients had a significantly longer progression-free survival than patients treated with interferon- $\alpha$ (11 vs 5 months, hazard ratio [HR]: 0.42, $P<0.001){ }^{1,2}$ In a randomized-controlled phase III trial of cytokine-refractory patients with advanced clear cell $\mathrm{RCC}$, sorafenib demonstrated prolonged progression-free survival relative to placebo 
Table I Summary of phase III randomized controlled trials of targeted therapies for advanced renal cell carcinoma

\begin{tabular}{|c|c|c|c|c|}
\hline Regimen & Sample size & Hazard ratio $(95 \% \mathrm{Cl})$ & PFS (absolute gain), months & Stable disease (\%) \\
\hline Everolimus vs placebo ${ }^{22,23}$ & 410 & $0.33(0.22-0.40)$ & 4.9 vs I.9 $(+3.0)(P<0.00 I)$ & 67 \\
\hline Sorafenib vs placebo 3,4 & 903 & $0.44(0.35-0.55)$ & 5.5 vs $2.8(+2.8)(P<0.0 \mathrm{I})$ & $74^{\mathrm{a}}$ \\
\hline Pazopanib vs placebo ${ }^{5}$ & 435 & $0.46(0.34-0.62)$ & 9.2 vs $4.2(+5.0)(P<0.00 \mathrm{I})$ & 38 \\
\hline Sunitinib vs interferon- $\alpha^{1,2}$ & 750 & $0.54(0.45-0.64)$ & II.0 vs $5.0(+6.0)(P<0.001)$ & 44 \\
\hline Bevacizumab + interferon- $\alpha$ vs interferon- $\alpha^{6}$ & 649 & $0.63(0.52-0.75)$ & 10.2 vs $5.4(+4.8)(P<0.001)$ & 46 \\
\hline Bevacizumab + interferon- $\alpha$ vs interferon- $\alpha^{7,27}$ & 732 & $0.7 \mathrm{I}(0.6 \mathrm{I}-0.83)$ & 8.5 vs $5.2(+3.3)(P<0.001)$ & $26^{\mathrm{b}}$ \\
\hline Temsirolimus vs interferon- $\alpha^{21}$ & 626 & $0.73(0.58-0.92)^{c}$ & 5.5 vs $3.1(+2.4)(P<0.00 I)$ & $32^{\mathrm{d}}$ \\
\hline
\end{tabular}

Notes: ${ }^{\mathrm{a}}$ Defined as disease that remained unchanged for $\leq 28$ days; ${ }^{\mathrm{b}} \mathrm{Defined}$ as objective response rate; ${ }^{\mathrm{C} H a z a r d}$ ratio for death; ${ }^{\mathrm{d} D e f i n e d ~ a s ~ o b j e c t i v e ~ r e s p o n s e ~ o r ~ s t a b l e ~}$ disease $\geq 24$ weeks.

Abbreviations: $95 \% \mathrm{Cl}$, 95\% confidence interval; PFS, progression-free survival.

(5.5 vs 2.8 months, HR: $0.44, P<0.01){ }^{3,4}$ Pazopanib, recently approved by the Food and Drug Administration (FDA), is a selective inhibitor of VEGFR-1, VEGFR-2, VEGFR-3, c-Kit, PDGFR- $\alpha$, and PDGFR- $\beta$. In a group of treatment-naïve and cytokine-pretreated patients, overall progression-free survival was 9.2 vs 4.2 months (HR: $0.46, P<0.001$ ), and 11.1 vs 2.8 months (HR: $0.40, P<0.001$ ), respectively. ${ }^{5}$ Bevacizumab, combined with interferon- $\alpha$, is a humanized monoclonal antibody against VEGF-A, which prevents VEGF-A stimulation of its receptor (VEGFR-2) on endothelial cells. Its efficacy was proven in 2 phase III trials in which, relative to interferon- $\alpha$ alone, progression-free survival was significantly longer: 10.2 vs 5.4 months, HR: $0.63, P<0.001^{6}$ and 8.5 vs 5.2 months, HR: $0.71, P<0.001{ }^{7}$

Despite the advent of VEGF/VEGFR-targeted therapy, virtually all patients ultimately develop resistance and experience disease progression. A large proportion of patients who fail first-line targeted therapy are treated with sequential therapy, with the intent of extending the clinical benefit beyond that of monotherapy. ${ }^{8}$ Prior to the advent of everolimus, the sequential use of therapies against the same or similar target was already commonly practiced. For example, among mRCC patients who failed first-line VEGF-targeted therapy, $33 \%$ of patients received second-line therapy. ${ }^{9}$ Of those, $88 \%$ received a second VEGF inhibitor and $11 \%$ received a second $\mathrm{mTOR}$ inhibitor. In a nonrandomized controlled study, the sequential use of sorafenib-sunitinib and sunitinib-sorafenib achieved stable disease in 51\% and $55 \%$ of patients, respectively, and a delay in time to progression in both strategies. ${ }^{10}$ These results were corroborated in other studies of similar design and indicate that despite the similar mechanisms of action of sorafenib and sunitinib, little cross-resistance exists. ${ }^{11-15}$

Although good response rates after sequential use of VEGF/VEGFR inhibition have been recorded, it was postulated that targeting of the mTOR pathway may possibly increase the chances of disease stabilization and prolong a patient's time of progression-free survival. Initial phase I studies had established a dosing schedule and the safety of everolimus ${ }^{16-18}$ and temsirolimus ${ }^{19,20}$ in patients with various solid tumor malignancies. Subsequently, the efficacy of mammalian target rapamycin (mTOR) targeting was proven within a first-line phase III trial of previously untreated and predominantly poor-risk $(74 \%) \mathrm{mRCC}$ patients $(\mathrm{n}=626){ }^{21}$ Treatment with temsirolimus alone significantly increased overall survival (10.9 vs 7.3 months, HR: $0.73, P=0.008$ ) and progression-free survival relative to interferon- $\alpha$ (5.5 vs 3.1 months, HR not reported, $P<0.001)$. The first-line success of mTOR inhibition was recently replicated in second line. Here, everolimus $(n=272)$ was compared with placebo $(n=138)$ in mRCC patients who had previously progressed on one of two prior anti-VEGF agents. ${ }^{22,23}$ Final results of that trial demonstrated that patients enrolled in the everolimus group had a significantly longer progression-free survival than those in the placebo group (4.9 vs 1.9 months, HR: 0.33, $P<0.001)$. The findings of that trial confirmed that clinical resistance to VEGF inhibitors does not imply clinical resistance to mTOR inhibitors. The current article examines the therapeutic benefit and the current role of everolimus in the management of patients with $\mathrm{mRCC}$.

\section{mTOR and $m$ TOR inhibitors mechanism}

The mTOR kinase integrates signals related to energy, nutrients, and oxygen, which determine whether the cell has the resources to grow and divide in response to growth factor stimulation. Positive signals permit mTOR to target S6 kinase 1, which triggers the ribosomal S6 protein and ribosomal synthesis, and eukaryotic translation initiation factor $4 \mathrm{E}$ binding protein, which then allows the transcription of the proteins that regulate cell growth, cell cycle progression, and cellular metabolism. The inappropriate activation 
of the mTOR signaling pathway in the pathogenesis of cancer often correlates with a more aggressive tumor and a worse prognosis.

mTOR inhibitors have a mechanism of action that is very distinct from that of established standard-of-care VEGF pathway inhibitors such as VEGF receptor tyrosine kinase inhibitors (sunitinib, sorafenib) and VEGF ligand antibodies (bevacizumab). Both everolimus and temsirolimus bind to an intracellular protein, FKBP-12, forming a complex that inhibits mTOR serine-threonine kinase signaling. The disruption of mTOR signaling components upstream of mTOR suppresses the production of proteins that regulate progression through the cell cycle and angiogenesis. From a clinical perspective, this inhibition is highly pertinent since unregulated angiogenesis is prominent in RCC.

\section{Clinical efficacy of everolimus}

The use of mTOR inhibitors in the setting of $\mathrm{mRCC}$ patients was confirmed in phase III trial that examined overall survival as a primary endpoint in patients treated with temsirolimus vs interferon- $\alpha$ vs a combination of temsirolimus and interferon- $\alpha{ }^{21}$ Enrolled patients were treatment-naïve, predominantly poor-risk (74\%), and of clear cell histological subtype $(80 \%)$. In that study, a statistically significant overall survival benefit was demonstrated with temsirolimus relative to interferon- $\alpha$ in primary analyses.

In contrast, everolimus was tested in patients with substantially different characteristics than the temsirolimus patient population. ${ }^{22}$ Its efficacy was first examined in an uncontrolled phase II trial of everolimus that enrolled 41 patients with a previous history of $\geq 1$ therapy regimen and no therapy within $\leq 4$ weeks or enrollment at a dose of $10 \mathrm{mg}$ daily through a 4 -week cycle. ${ }^{24}$ The results showed a high proportion of patients with disease stabilization ( $\geq 3$ months: $74.2 \%, \geq 6$ months: $58.1 \%$ ). Subsequently, a randomized phase III trial was conducted of everolimus vs placebo for treatment of $\mathrm{MRCC}$ in patients whose disease had progressed on treatment with VEGFR tyrosine kinase inhibitors. $^{22}$ Those treated previously with sunitinib represented $46 \%$ of the sample population vs $28 \%$ with sorafenib vs $26 \%$ for both sunitinib and sorafenib. Most patients had favorable or intermediate-risk factors $(29 \%$ and $56 \%$, respectively). Efficacy analyses revealed that disease stabilization was twice as high in everolimus relative to placebo-treated patients (67 vs 32\%). Final interim analysis revealed a median progression-free survival of 4.9 vs 1.9 months for everolimus vs placebo, respectively (HR: $0.33, P<0.001) .{ }^{23}$ Due to a significant number of patients $(80 \%)$ who crossed over during the study period, a difference in overall survival was not detected (HR: 0.83, $P=0.2$ ). To correct for the crossover effect, the rank-preserving structural failure time model was used and demonstrated that everolimus reduced the risk of death by 1.9 -fold $(P<0.01){ }^{23}$

Most commonly reported adverse events of any grade associated with everolimus were stomatitis (44\%) and infections (including pneumonia, aspergillosis, candidiasis, and sepsis: $37 \%) .{ }^{23}$ Most commonly reported grade 3 or 4 toxicities were infections $(10 \%)$, stomatitis $(5 \%)$, fatigue $(5 \%)$, and dyspnea (7\%). Decreasing hemoglobin (92\%) and lymphocytes (51\%), as well as increasing cholesterol (77\%) and triglycerides $(73 \%)$ were among the most commonly reported laboratory abnormalities of any grade. Decreasing hemoglobin (13\%) and lymphocytes (18\%), and glucose augmentation (16\%) were the most commonly observed grade 3 or 4 adverse events.

Everolimus received FDA approval, specifically for treatment of patients with advanced RCC after failure of treatment with sunitinib or sorafenib. Moreover, it was given the category 1 level of recommendation by the National Comprehensive Cancer Network guidelines for second-line treatment of patients with advanced RCC after failure of tyrosine kinase inhibitor-therapy. ${ }^{25}$

\section{What is important to consider about everolimus in second or subsequent line for $\mathrm{mRCC}$}

1. The everolimus data represent the first placebo-controlled report of efficacy of sequential targeted therapies in mRCC. ${ }^{22}$ Relative to placebo, patients receiving everolimus were $67 \%$ less likely to progress, as evidenced by the HR of $0.33(P<0.001) .{ }^{22}$ Thus, the beneficial effect of everolimus is the strongest among all other molecules tested in phase III trials, in terms of progression-free survival. For example, in first-line therapy, sunitinib demonstrated a HR of $0.54(P<0.001)$ compared with interferon- $\alpha .^{1}$ The efficacy of other molecules ranged from 0.44 to 0.73 (Figure 1).

Taken together, the everolimus data indicate an HR (0.33) that substantially surpasses all other existing targeted therapies, in the context of progression-free survival. Moreover, despite the aggressive nature of the examined mRCC population, up to 3.5 months of progression-free survival benefit relative to placebo may be expected with second-line therapy. ${ }^{26}$

2. The control group of the everolimus study provides an excellent opportunity to ascertain the aggressiveness of the 


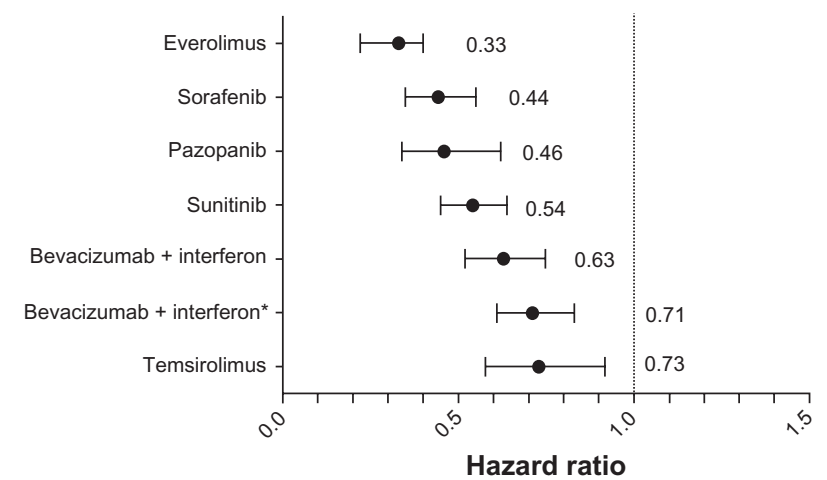

Figure I Hazard ratios of progression-free survival data from phase III randomized controlled trials. Lines represent $95 \%$ confidence intervals. ${ }^{27}$ The dotted line represents the reference group.

Note: *Based on the CALGB phase III trial.

population that was studied. Specifically, the median progression-free survival in the placebo arm was 1.9 months. This length of progression-free survival strongly contrasts with the length of progression-free survival recorded in placebo groups of the sunitinib ${ }^{2}$ or pazopanib ${ }^{5}$ trials, in which, respectively, 5.0 and 4.2 months progression-free survival durations were recorded.

A substantially more aggressive behavior is expected in pretreated patients, as was the case in the everolimus study. It is of note that the median progression-free survival in the interferon- $\alpha$ arm of the temsirolimus phase III study that focused on poor-risk patients was 3.1 months. ${ }^{21}$ This estimate indicates that the everolimus study ${ }^{22}$ patients had an even more aggressive disease than poor-risk patients examined in the temsirolimus phase III trial.

Taken together, these observations indicate that the impressive benefit recorded in the everolimus study was recorded despite it being tested in the cohort of patients with the most aggressive natural history of the disease ever examined in a phase III $\mathrm{mRCC}$ design.

3. It is also highly noteworthy that the efficacy of everolimus differed according to the number of previous targeted therapies. In a subanalysis, individuals who received only 1 anti-VEGF therapy (sunitinib or sorafenib) were compared with patients who received both sunitinib and sorafenib. ${ }^{26}$ Patients who received only 1 prior anti-VEGF agent gained 3.5 months progression-free survival compared with the 3.0 months gained by placebo patients in the overall population in which everolimus was tested. Moreover, it was revealed that gain in progression-free survival of patients previously treated with sorafenib only (3.1 months, HR: $0.25, P<0.001$ ) was greater than in patients previously treated with sunitinib only (2.1 months, HR: $0.34, P<0.001){ }^{23}$
The interpretation of these data in the context of clinical practice where the place of everolimus is mainly in second or subsequent lines indicates that more observable progression-free survival benefits than those recorded for the entire population should be expected.

4. It is equally important to examine the everolimus data in the context of multiple previous treatment lines. Since 26\% of patients in the everolimus study received more than one anti-VEGF therapy, this subset of patients is the only available evidence that a benefit beyond second line is possible in a randomized-placebo controlled design.

5. The progression-free survival benefit in the everolimus arm of the study needs to be considered in the light of health-related quality-of-life and toxicity data - despite receiving treatment with everolimus, patients' healthrelated quality of life remained unaffected. ${ }^{22}$

Stable health-related quality of life is important to consider in the light of everolimus toxicity. Despite a low but statistically significant increase in grade 3 and 4 toxicities, patients exposed to everolimus did not report health-related quality-of-life deterioration. These findings indicate that everolimus toxicity is well tolerated and that the therapeutic benefits of everolimus are associated with sustained health-related quality of life.

6. Despite its benefits for progression-free survival, maintenance of health-related quality of life, and rarity of grade 3 or 4 toxicities, everolimus data are undermined by absence of overall survival benefits. It is noteworthy for mRCC targeted therapies, overall survival benefit was recorded only in the temsirolimus trial, ${ }^{21}$ which corresponds to 1 out of 7 phase III mRCC trials. 2,3,5,6,21,22,27

It is important to note that the overall survival benefit for temsirolimus was demonstrated within a cohort of patients with highly aggressive mRCC. The natural history of $\mathrm{mRCC}$ in these individuals limited the potential for sequential therapy. Moreover, at the time of the temsirolimus trial, the notion of sequential therapy did not yet exist. Finally, no crossovers occurred from interferon- $\alpha$ or interferon- $\alpha$ plus temsirolimus into the temsirolimus-only arm. In consequence, no contamination of either progression-free survival or overall survival could have occurred in that study.

Unlike in the study evaluating temsirolimus, ethical and efficacy considerations at the time of the everolimus study design and conduct required crossover of the placebo arm. ${ }^{22}$ Indeed, $81 \%$ of placebo patients switched to the everolimus group. This measure diluted the effect of everolimus on survival and expectedly resulted in a 
virtually null effect. This phenomenon was previously reported in the phase III trial of sunitinib ${ }^{1}$ and in the phase III trial of sorafenib. ${ }^{4}$ In both settings, crossover significantly undermined the therapeutic effect of both molecules, especially the effect on overall survival.

Therefore, everolimus is not the first molecule for which lack of statistically significant overall survival benefit represents a limitation, and certainly will not be the last. In the context of sequential therapies, this limitation will affect all future studies in the field of mRCC. Additionally, in all future studies, subsequent lines of therapy will invariably contaminate overall survival. In consequence, it could be postulated that valid assessment of overall survival is not possible except for the terminal sequence of best supportive care, and death.

7. Lack of valid overall survival data will invariably affect the assessment of cost-benefit of novel therapies. This limitation affects everolimus. The calculation of this costbenefit ratio for novel agents requires overall survival data, where cost per quality-adjusted life-years needs to be defined for comparison purposes. Unfortunately, absence of real-life overall survival data in everolimustreated patients precludes direct calculation of such a metric. To obviate this problem, a Markov-cost-utility model was used in the evidence review group report to project 4 distinct health states: stable disease without adverse events, stable disease with adverse events, progressive disease, and death. ${ }^{28}$ The use of such modeling may introduce significant bias that under- or overestimates true overall survival. Unfortunately, the magnitude of such bias cannot be quantified. In consequence, such model predictions and the resulting quality-adjusted lifeyears cost estimation is hypothetical at best.

In consequence, it may be impossible to quantify the cost of quality-adjusted life-years for most sequential therapies in $\mathrm{mRCC}$, using reliable and valid estimates. Under this premise, the cost-benefit of sequential therapies may not be directly comparable with that of chemotherapeutic agents for which overall survival estimates can be calculated with known validity and reliability. In the context of everolimus, lack of such data does not represent a limitation, since there is no alternative sequential agent with phase III proven efficacy.

8. Finally, it remains unclear whether either of these individual mTOR inhibitors, temsirolimus or everolimus, have any advantage over one another as they have not been compared head-to-head. In the setting of $\mathrm{mRCC}$, both agents were tested on very different populations and are advocated under very different situations - temsirolimus for patients with predominantly poor-risk factors and everolimus for patients who had previously progressed on at least 1 targeted therapy. Furthermore, one may also consider the use of sirolimus in the treatment management of mRCC patients. However, data on this agent are still limited. ${ }^{29}$

\section{Ongoing trials of everolimus in RCC}

Despite its established role in sequential therapy for $\mathrm{mRCC}$, the efficacy of everolimus is unknown in first line. The ongoing RECORD-3 phase II study seeks to compare everolimus in first line followed by second-line sunitinib vs sunitinib followed by everolimus [NCT00903175]. The study attempts to identify the ideal sequence for the administration of sunitinib and everolimus. Moreover, it will provide a contemporary assessment of everolimus efficacy in second line. Despite its goal, it is questionable that evidence from a phase II trial will displace sunitinib as first-line standard of care that was established with phase III data. Two other phase II studies compare the efficacy of everolimus and sunitinib in patients with nonclear-cell histological subtype [NCT01108445] or in patients with specifically papillary RCC [NCT00688753]. The results from these studies will require comparison with temsirolimus phase III data in the subset of individuals with nonclear-cell histological subtype. Two ongoing phase III studies are assessing the benefit, if any, of everolimus and bevacizumab combination in second-line therapy [NCT00719264, NCT01198158]. Finally, efficacy of everolimus in both the neoadjuvant and adjuvant settings will be investigated [NCT01120249, NCT01107509].

\section{Conclusion}

Everolimus is currently the only agent with a proven progression-free survival improvement in a randomized phase III study on patients who progressed on VEGFR-tyrosine kinase inhibitor. Moreover, it demonstrated the strongest HR for progression-free survival ever recorded among all mRCC phase III trials, despite it being tested in a population with the most aggressive natural history of the disease. Due to its safety and benefit, it is considered a therapeutic standard of care for patients who previously failed VEGFR treatment. Moreover, it represents the first placebo-controlled evidence of efficacy for a sequentially used targeted agent. Future studies may further shed light on the most optimal timing and sequence of everolimus compared with other available targeted agents.

\section{Disclosure}

The authors declare no conflicts of interest. 


\section{References}

1. Motzer RJ, Hutson TE, Tomczak P, et al. Overall survival and updated results for sunitinib compared with interferon alfa in patients with metastatic renal cell carcinoma. J Clin Oncol. 2009;27(22):3584-3590.

2. Motzer RJ, Hutson TE, Tomczak P, et al. Sunitinib versus interferon alfa in metastatic renal-cell carcinoma. $N$ Engl J Med. 2007;356(2):115-124.

3. Escudier B, Eisen T, Stadler WM, et al. Sorafenib in advanced clear-cell renal-cell carcinoma. N Engl J Med. 2007;356(2):125-134.

4. Escudier B, Eisen T, Stadler WM, et al. Sorafenib for treatment of renal cell carcinoma: final efficacy and safety results of the phase III treatment approaches in renal cancer global evaluation trial. J Clin Oncol. 2009;27(20):3312-3318.

5. Sternberg C, Davis I, Mardiak J, et al. Pazopanib in locally advanced or metastatic renal cell carcinoma: results of a randomized phase III trial. J Clin Oncol. 2010;28(6):1061-1068.

6. Escudier B, Pluzanska A, Koralewski P, et al. Bevacizumab plus interferon alfa-2a for treatment of metastatic renal cell carcinoma: a randomised, double-blind phase III trial. Lancet. 2007;370(9605):2103-2111.

7. Rini BI, Halabi S, Rosenberg JE, et al. Phase III trial of bevacizumab plus interferon alfa versus interferon alfa monotherapy in patients with metastatic renal cell carcinoma: final results of CALGB 90206. J Clin Oncol. 2010;28(13):2137-2143.

8. Escudier B, Goupil MG, Massard C, Fizazi K. Sequential therapy in renal cell carcinoma. Cancer. 2009;115(10 Suppl):2321-2326.

9. Vickers MM, Choueiri TK, Rogers M, et al. Clinical outcome in metastatic renal cell carcinoma patients after failure of initial vascular endothelial growth factor-targeted therapy. Urology. 2010;76(2): 430-434.

10. Sablin MP, Negrier S, Ravaud A, et al. Sequential sorafenib and sunitinib for renal cell carcinoma. J Urol. 2010;182(1):29-34.

11. Garcia JA, Hutson TE, Elson P, et al. Sorafenib in patients with metastatic renal cell carcinoma refractory to either sunitinib or bevacizumab. Cancer. 2010;116(23):5383-5390.

12. Eichelberg C, Heuer R, Chun FK, et al. Sequential use of the tyrosine kinase inhibitors sorafenib and sunitinib in metastatic renal cell carcinoma: a retrospective outcome analysis. Eur Urol. 2008; 54(6):1373-1378

13. Dudek AZ, Zolnierek J, Dham A, Lindgren BR, Szczylik C. Sequential therapy with sorafenib and sunitinib in renal cell carcinoma. Cancer. 2009; 115(1):61-67.

14. Di Lorenzo G, Cartenì G, Autorino R, et al. Phase II study of sorafenib in patients with sunitinib-refractory metastatic renal cell cancer. $J$ Clin Oncol. 2009;27(27):4469-4474.

15. Di Lorenzo G, Buonerba C, Federico P, et al. Third-line sorafenib after sequential therapy with sunitinib and mTOR inhibitors in metastatic renal cell carcinoma. Eur Urol. In press.

16. Tanaka C, O'Reilly T, Kovarik J, et al. Identifying optimal biologic doses of everolimus (RAD001) in patients with cancer based on the modeling of preclinical and clinical pharmacokinetic and pharmacodynamic data. J Clin Oncol. 2008;26(10):1596.
17. Tabernero J, Rojo F, Calvo E, et al. Dose-and schedule-dependent inhibition of the mammalian target of rapamycin pathway with everolimus: a phase I tumor pharmacodynamic study in patients with advanced solid tumors. J Clin Oncol. 2008;26(10):1603.

18. O'donnell A, Faivre S, Burris H, et al. Phase I pharmacokinetic and pharmacodynamic study of the oral mammalian target of rapamycin inhibitor everolimus in patients with advanced solid tumors. J Clin Oncol. 2008;26(10):1588.

19. Raymond E, Alexandre J, Faivre S, et al. Safety and pharmacokinetics of escalated doses of weekly intravenous infusion of CCI-779, a novel mTOR inhibitor, in patients with cancer. $J$ Clin Oncol. 2004;22(12):2336.

20. Hidalgo M, Buckner J, Erlichman C, et al. A phase I and pharmacokinetic study of temsirolimus (CCI-779) administered intravenously daily for 5 days every 2 weeks to patients with advanced cancer. Clin Cancer Res. 2006;12(19):5755.

21. Hudes G, Carducci M, Tomczak P, et al. Temsirolimus, interferon alfa, or both for advanced renal-cell carcinoma. $N$ Engl J Med. 2007;356(22):2271-2281.

22. Motzer RJ, Escudier B, Oudard S, et al. Efficacy of everolimus in advanced renal cell carcinoma: a double-blind, randomised, placebocontrolled phase III trial. Lancet. 2008;372(9637):449-456.

23. Motzer RJ, Escudier B, Oudard S, et al. Phase 3 trial of everolimus for metastatic renal cell carcinoma : final results and analysis of prognostic factors. Cancer. 2010;116(18):4256-4265.

24. Amato RJ, Jac J, Giessinger S, Saxena S, Willis JP. A phase 2 study with a daily regimen of the oral mTOR inhibitor RAD001 (everolimus) in patients with metastatic clear cell renal cell cancer. Cancer. 2009;115(11):2438-2446.

25. NCCN. Kidney Cancer. NCCN, 2010. http://www.nccn.org/professionals/ physician_gls/f_guidelines.asp. 2009. Accessed December 2010.

26. Figlin RA, Calvo E, Motzer T, et al. Everolimus in metastatic renal cell carcinoma (mRCC): Subgroup analysis of patients (pts) with one versus two prior vascular endothelial growth factor receptor tyrosine kinase inhibitor (VEGFR-TKI) therapies enrolled in the phase III RECORD-1 study. Paper presented at: 2011 Genitourinary Cancers Symposium; February 17-19, 2011; Orlando, FL. J Clin Oncol. 2011;29(Suppl 7: abstract 304).

27. Rini BI, Halabi S, Rosenberg JE, et al. Bevacizumab plus interferon alfa compared with interferon alfa monotherapy in patients with metastatic renal cell carcinoma: CALGB 90206. J Clin Oncol. 2008;26(33):5422-5428.

28. Pitt M, Crathorne L, Moxham T, Bond M, Hyde C. Everolimus for the second-line treatment of advanced and/or metastatic renal cell cancer: a critique of the submission from Novartis. Health Technol Assess. 2010;14(Suppl 2):41-46.

29. Flaig TW, Costa LJ, Gustafson DL, et al. Safety and efficacy of the combination of erlotinib and sirolimus for the treatment of metastatic renal cell carcinoma after failure of sunitinib or sorafenib. Br J Cancer. 2010;103(6):796-801.
Open Access Journal of Urology

\section{Publish your work in this journal}

The Open Access Journal of Urology is an international, peer-reviewed, open access journal publishing original research, reports, editorials, reviews and commentaries on all aspects of adult and pediatric urology in the clinic and laboratory including the following topics: Pathology, pathophysiology of urological disease; Investigation and treatment of

\section{Dovepress}

urological disease; Pharmacology of drugs used for the treatment of urological disease. The manuscript management system is completely online and includes a very quick and fair peer-review system, which is all easy to use. Visit http://www.dovepress.com/testimonials.php to read real quotes from published authors. 